\section{Delayed presentation of con- genital diaphragmatic hernia masquerading as pulmonary tuberculosis in a 4-year-old girl}

\author{
Abdullahi Salisu Shaaibu, ${ }^{1}$ \\ Aliyu Mohammed El-Habeeb, ${ }^{2}$ \\ Idris Mohammed Mustapha, ${ }^{2}$ \\ Abdulsalam Usman Danjaji ${ }^{3}$ \\ ${ }^{1}$ Department of Radiology, Aminu Kano \\ Teaching Hospital, Kano State; \\ 2Department of Radiology, Federal \\ Medical Center Katsina, Katsina State; \\ ${ }^{3}$ Department of Radiology, Murtala \\ Muhd Specialist Hospital, Kano State, \\ Nigeria
}

\begin{abstract}
Diaphragmatic hernias are defined as congenital or acquired defect in the diaphragm. It is said to be the most common intra-thoracic fetal anomaly with an incidence of 1 in 2200 to 3000 live births and male to female ratio of $2: 1$. The unusual and misleading presentation of this condition which would cause an unnecessary delay in diagnosis and all the attendant consequences (such as intestinal obstruction) is hereby presented. The role of plain chest Xray which is cheap and readily available in salvaging the confusing clinical scenario has been emphasized. Late presenting $\mathrm{CDH}$ should be factored in the differential diagnosis of any child with chronic cough. Delay in diagnosis increases chances of complication such as chronic lung diseases and gastro-esophageal reflux, later in adulthood.
\end{abstract}

\section{Introduction}

Diaphragmatic hernias are defined as congenital or acquired defect in the diaphragm. ${ }^{1}$ It is said to be the most common intra-thoracic fetal anomaly with an incidence of 1 in 2,200 to 3,000 live births and male to female ratio of $2: 1 .^{2}$ Eightyfour $(84) \%$ are left-sided, $13 \%$ are rightsided and $2 \%$ bilateral. $^{3}$

It is caused by delayed fusion of diaphragm or premature return of bowel from its herniated position within the umbilical coelom. ${ }^{2}$ It may also result from an insult that inhibits or delays normal migration of the gut and closure of the diaphragm between $8^{\text {th }}$ and $12^{\text {th }}$ week of embryogenesis. ${ }^{2}$

There are two main types of congenital diaphragmatic hernia (CDH); the much commoner Bochdalek hernia (85-90\%) and Morgagni hernia (3-5\%). ${ }^{2}$ Bochdalek hernia is usually located posterior-laterally and usually present in infancy, while Morgagni hernia is smaller, usually located anteriormedially and presents later, in older children.

Clinical presentation depends on the side, severity and herniated organ(s) involved. Most congenital diaphragmatic hernias are detected either soon after birth or on antenatal ultrasound. ${ }^{4}$ Mortality is predominantly due to development of pulmonary hypoplasia, which is thought to be due to mass effect on the developing lung. Right sided hernias are frequently fatal. ${ }^{2}$ Small bowel is the most frequently affected $(90 \%)$, followed by stomach $(60 \%)$, large bowel (56\%), spleen (54\%), pancreas $(24 \%)$ and kidney ((12\%). Adrenal gland, liver and gallbladder are least affected. ${ }^{2}$ Patients may present with scaphoid abdomen and respiratory distress in neonatal period. Bowel sounds may be auscultated in the chest. There may be contralateral shift of mediastinum and the heart. Nasogastric tube may be seen entering intra-thoracic stomach when passed under fluoroscopic control. ${ }^{2}$

The unusual and misleading presentation of congenital diaphragmatic hernia with a potential of life-threatening complications (such as intestinal obstruction and perforation), leading to delay in its diagnosis in a 4-year-old child, is hereby presented. This case report further emphasizes the role of plain chest $\mathrm{x}$-ray (which is cheap and readily available) in addressing such confusing clinical scenario.

\section{Case Report}

$\mathrm{ZK}$ is a 4-year-old school girl who presented to outpatient department on account of chronic cough for about 8 months prior to presentation. The cough was said to be unproductive, worse when patient is asleep. There is associated history of epigastric discomfort and refusal to eat. No chest pain, fever or night sweat. No history of contact with chronically coughing adult. No history of delayed developmental milestone. No previous history of trauma to the chest or abdomen. Patient said to have been fully immunized. Patient was self-medicated repeatedly at home with anti-tussives for her cough symptoms but no remarkable improvement was observed and hence brought to hospital.

On examination, she was underweight, afebrile with a scaphoid abdomen. Chest examination was not detailed initially and was reported as normal. Her vital signs
Correspondence: Abdullahi Salisu Shaaibu, Department of Radiology, Aminu Kano Teaching Hospital, PMB 3452, Kano, Kano State, Nigeria.

Tel.: +2348032878364.

E-mail: drsharubutu@gmail.com

Key words: congenital, diaphragmatic hernia, pulmonary tuberculosis.

Contributions: the authors contributed equally.

Conflict of interest: the authors declare no potential conflict of interest.

Funding: none.

Received for publication: 28 September 2018. Accepted for publication: 23 April 2019.

This work is licensed under a Creative Commons Attribution NonCommercial 4.0 License (CC BY-NC 4.0).

${ }^{\circ}$ Copyright: the Author(s), 2019

Licensee PAGEPress, Italy

Pyramid Journal of Medicine 2019; 2:26

doi:10.4081/pjm.2019.26

were essentially normal. Chest x-ray was requested for possible respiratory tract infection (pulmonary tuberculosis). The radiograph revealed opacification of the right hemithorax, including the retrosternal area, by suspected loops of bowel (Figures 1 and 2). There is slight mediastinal shift to the left. The left lung, heart and the rib cage are within normal limits. Based on the radiographic findings of the chest, a diagnosis of possible congenital diaphragmatic hernia with late presentation was made.

A single contrast barium enema was performed which demonstrated herniation of part of colon into the right hemithorax (Figure 3). A diagnosis of right sided congenital diaphragmatic hernia, most likely Morgagni type with delayed presentation was made. Patient was later booked for surgery where the diagnosis was further confirmed and the hernial defect was successfully repaired. A post op chest radiograph was later obtained which appeared normal (Figure 4).

\section{Discussion and Conclusions}

Congenital diaphragmatic hernia $(\mathrm{CDH})$ may vary in size and occurs in various portions of diaphragm. Bochdalek hernia is a posterior-lateral defect caused by mal-development/defective fusion of the cephalic fold of pleuroperitoneal mem- 
branes. It has an incidence of about 1 in 2200 to 12,500 live births. It is located on the left in $80 \%$ of cases, to the right in $15 \%$ and bilateral in $5 \% .^{2}$ Morgagni hernia on the other hand, constitutes $3-5 \%$ of $\mathrm{CDHs}$ and occurs through the anterior-medial parasternal defect (space of Larrey), located between the ribs and sternum. It is caused by maldevelopment of septum transversum. It has an incidence of about 1 in 4,800 live births and commoner among male child. ${ }^{2}$

The presentation of $\mathrm{CDH}$ beyond neonatal period is extremely varied, and may be associated with misleading clinical and radiologic assesments. ${ }^{5}$ Misdiagnosis is a distinct possibility, with the risk of serious morbidity and mortality. ${ }^{5}$ The late presentation of congenital diaphragmatic hernia was considered rare, but an increasing number of publications indicate that up to a quarter of patients are diagnosed after the neonatal period. ${ }^{6}$ This appears to be the case with this index patient who was apparently asymptomatic until her third year.

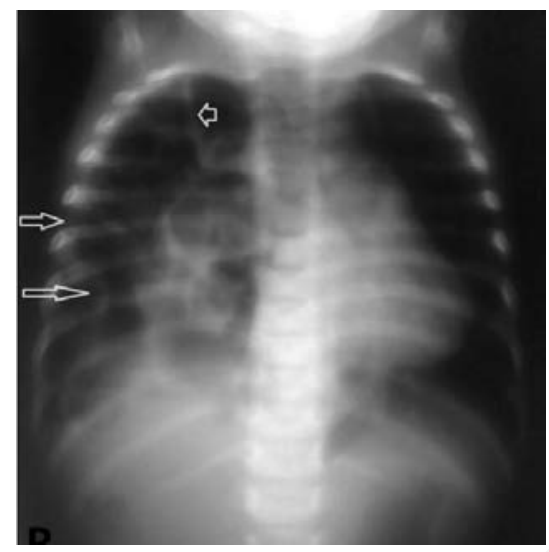

Figure 1. Frontal chest radiograph showing herniated bowel loops in the right hemithorax (arrows).

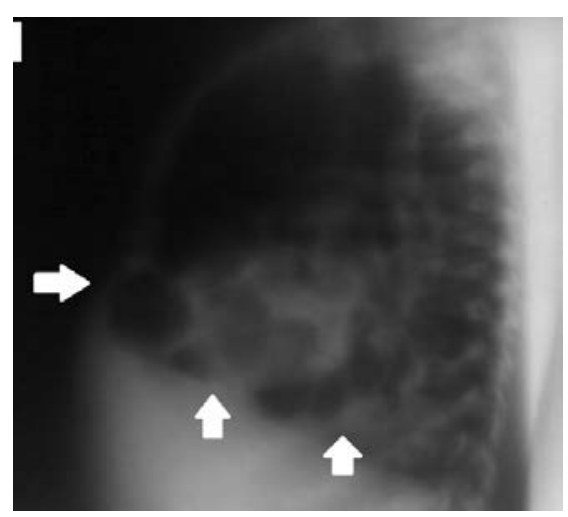

Figure 2. Lateral projection showing herniated bowel loops predominantly in the retrosternal area (arrows).
Patients present with a wide variety of gastrointestinal and respiratory symptoms, but at least a quarter will be asymptomatic, and are often diagnosed incidentally. ${ }^{6}$ The symptoms include epigastric discomfort, chronic cough, chocking, dyspnea and in severe cases, respiratory distress and cyanosis. ${ }^{2}$ A large multicenter study by Kitano et al., ${ }^{7}$ concluded that presenting symptoms of late-onset $\mathrm{CDH}$ can be respiratory or gastrointestinal, but presentation with gastrointestinal problems was more common in left-sided hernias, whereas respiratory symptoms predominated in rightsided lesions. Their finding is in conformity with that of the index case, which happened to have a right sided hernia and presented with a respiratory symptom (chronic cough).

There may be associated anomalies in $20 \%$ of live born and in $90 \%$ of stillborn fetuses. These anomalies include CNS (neural tube defects), CVS, GIT (particular-

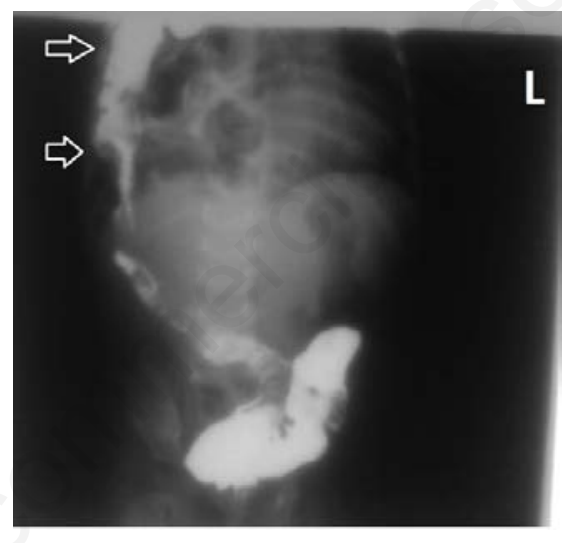

Figure 3. Barium enema showing continuity of colon in the abdomen with suspected loops in the chest, thus, confirming large bowel herniation into the right hemithorax.

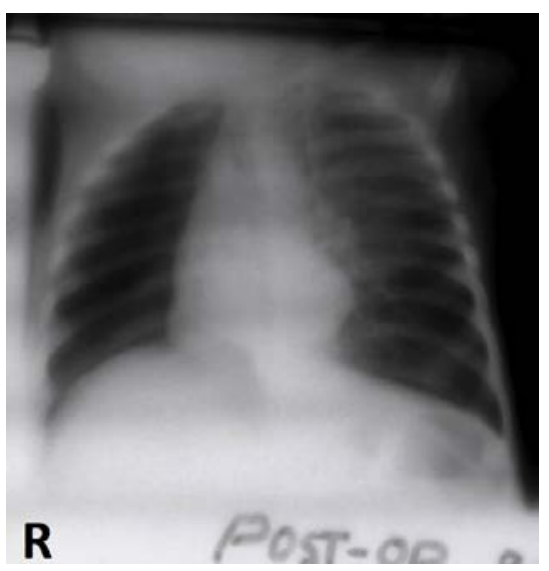

Figure 4. Normal post-operative frontal chest radiograph after successful repair. ly malrotation and omphalocele), chromosomal abnormality and intra uterine growth retardation. ${ }^{2}$ None of these anomalies were demonstrated in the index case.

Radiological investigations that are essential in the diagnosis include obstetric ultrasound scan, chest radiograph, barium studies and magnetic resonance imaging (MRI). Prenatal USS may show complex (cystic and solid) mass in the fetal chest, reduced abdominal circumference and intra-thoracic herniation of the liver, among other features ${ }^{4}$. It may show indirect signs, such as hydramnious. ${ }^{4}$ The observed-toexpected lung-to-head ratio (O/E LHR) may be calculated, and correlates with the degree of pulmonary hypoplasia. The ratio calculation is of prognostic significance ${ }^{3}$. Chest radiograph may show indistinct diaphragm with opacification of part of or all the hemithorax. The suspected herniated bowel loop can be confirmed by demonstrating barium-coated stomach or intestine in the thorax, following barium meal or enema studies. MRI may be helpful in further assessing the hernia and any associated pulmonary hypoplasia. ${ }^{3}$

Treatment is mainly surgery, which should be done as emergency especially in younger children to avoid possible occurrence of life-threatening complications such as respiratory distress and strangulated intestinal obstruction.

Late-presenting CDHs are usually repaired through a laparotomy, as withdrawal of the contents downward is considered easier than pushing them down. Also, associated intestinal anomalies can be conveniently dealt with. Malrotation should be excluded preoperatively by a barium meal and follow-through should a transthoracic approach be considered. ${ }^{5}$

The prognosis of right-sided hernias seems to be worse than left-sided ones. . $^{2,8}$ Restrictive and obstructive lung diseases have been reported in $\mathrm{CDH}$ survivors many years after operation and diaphragmatic rigidity and thoracic deformities can also play a minor role in chronic lung disease. ${ }^{8}$ Other sequelae such as gastro esophageal reflux disease are possible in this particular group of patients. These, and the frequent associated malformations, require longterm follow-up and permanent support. ${ }^{8}$

\section{References}

1. Radiopaedia.org. Diaphragmatic hernia. Available from: www.radiopaedia. org/articles/diaphragmatic-hernia. Accessed on 02/02/16.

2. Wolfgang D, ed. Chest Disorders. In: Radiology Review Manual. 6th Ed. 
Philadelphia: Lippincot Williams \& Wilkins; 2007. pp 498-499.

3. Gucciardo L, Deprest J, Done' E, et al. Prediction of outcome in isolated congenital diaphragmatic hernia and its consequences for fetal therapy. Best Pract Res Clin Obstet Gynaecol 2008;22:123-38.

4. Taylor GA, Atalabi OM, Estroff JA.
Imaging of congenital diaphragmatic hernias. Pediatr Radiol 2009;39:1-16.

5. Elhalaby EA, Sikeena MA. Delayed presentation of congenital diaphragmatic hernia. Pediatr Surg Int 2002; 18:4805.

6. Bhandari BB, Basnet RB. Late Presentations of Congenital Diaphragmatic Hernia. J Nepal Health
Res Counc 2011 Dec 18.

7. Congenital Diaphragmatic Hernia Study Group. Late-presenting congenital diaphragmatic hernia. J Pediat Surg 2005;40:1839-43.

8. Tovar JA. Congenital diaphragmatic hernia. Orphan J Rare Dis 2012;7:1. 